\title{
Numerical Experiments on String Cosmology
}

\author{
Mairi Sakellariadou 円 \\ Institut für Theoretische Physik \\ der Universität Zürich, Winterthurerstr. 190, \\ CH-8057 Zürich, Switzerland
}

\begin{abstract}
We investigate some classical aspects of fundamental strings via numerical experiments. In particular, we study the thermodynamics of a string network within a toroidal universe, as a function of string energy density and space dimensionality. We find that when the energy density of the system is low, the dominant part of the string is in the form of closed loops of the shortest allowed size, which correspond to the momentum string modes. At a certain critical energy density corresponding to the Hagedorn temperature, the system undergoes a phase transition characterized by the formation of very long loops, winding a number of times around the torus. These loops correspond to the winding string modes. As the energy density is increased, all the extra energy goes into these long strings. We then study the lifetime of winding modes as a function of the space densionality. We find that in the low-energy density regime, long winding strings decay only if the space dimensionality of the toroidal universe is equal to 3 . This finding supports the proposed cosmological scenario by Brandenberger and Vafa, which attempts to explain the space dimensionality and to avoid the initial singularity by means of string theory.
\end{abstract}

PACS numbers: 98.80.Cq, 98.80.Bp, 05.90.+m

\section{Introduction}

One of the main questions facing string theory in many potentially exciting applications is the understanding of the behaviour of strings in extreme conditions of high temperatures and high densities. One would expect these domains to be achieved

\footnotetext{
${ }^{1}$ Current address: Université de Genève, Département de Physique Théorique, 24 quai Ernest Anserment, CH-1211 Genève, Switzerland; e-mail address: mairi@karystos.unige.ch
} 
in the context of the eraly universe. If string theory is a theory unifying matter and gravity, then one should expect the evolution of the universe, in particular around the Planck scale, to deviate from its description according to standard cosmology. Our homogeneous, isotropic and almost flat universe, should be the remnant of the evolution of the universe as described by string theory. Thus we need to do string cosmology, in other words, to study string behaviour at high energy density and temperature. In addition, since stringy effects become important at an energy scale much beyond the one we can probe in high energy scattering experiments, obtaining currently measurable cosmological consequences of string theory will allow us to get a confrontation between string theory and experiments.

However the main difficulty in using string theory in such a context lies in the fact that one would have to know about non-perturbative aspects of strings, which we do not have much understanding of at the present. It would thus be desirable to have a model for strings which one can trust in such domains. In this paper we explore one such model and its physical implications.

Usually when we discuss string theory we immediately turn to the quantum theory. We consider first quantized strings consisting of the graviton and all the infinitely many massive stringy excitations and talk about their interactions etc. This was indeed the main motivation for studying string theory in the first place, i.e., the fact that it leads to a consistent quantum theory of gravity. However, one expects that many properties of strings should be dominated by classical aspects of strings. In this context one could deal with all the modes of strings at once which are in a coherent state and behave in a classical way. Our aim is to study some classical aspects that strings have. There are two postulates we are going to make which we find reasonable:

1-Classical strings are simply described by the Nambu [1] - Goto [2] action and thus behave very much like cosmic strings. Moreover it will be assumed that 
whenever two strings meet at a point they intercommute with a certain probability.

2-There is a maximum energy density for strings which one can identify with the Planck density.

The first postulate is more or less the definition of classical strings modulo the condition for intercommutation when they meet, which is there to mimick string interactions. The second postulate is intuitively what one would expect but is harder to give a rigorous justification for. In a sense it is in accord with the intuition that there can be at most one string per Planck volume and that we cannot put infinitely many strings squeezed into a small region of space. At any rate this will be our assumption in this paper. Note that a particular consequence of this assumption is that the only degree of freedom for strings with the maximum density is how they are connected. The connectivity of strings becomes the relevant question near this maximum density.

Luckily both of these postulates are realized in the context of cosmic string simulations on a lattice [3], [4], which one can thus use in the context of fundamental strings.

Here, the aim is to study via numerical experiments the thermal properties of strings and equilibration process for strings, and in particular its dimension dependence. One motivation for this study is to test the model proposed in Ref. [5] for string cosmology. That model, which takes the space to be a $D$-dimensional torus, avoids the singularity of Friedmann-Robertson-Walker cosmology by a stringy method which is the well known $L \mapsto 1 / L$ duality of strings [6], stating that large and small tori are equivalent. It was further suggested that the dynamics dictating the evolution of the universe should be modified in the context of string theory from Einstein's equations in order for it to be consistent with this small-large duality. Moreover, it was suggested there that if long strings do not decay, then the expansion of the universe may stop as it would otherwise create perpetual energy. This 
latter point, which was postulated there, together with the modification needed to bring Einstein's equation in line with stringy duality, was shown [7] to be the consequence of the existence of the dilaton in string theory. It was further suggested in Ref. [5] that the macroscopic dimension of space may be 3, because long strings in higher dimensions have a harder time chopping themselves up to short strings, which combined with the equations of string evolution will be consistent with expansion only for space dimensions less than or equal to three? The main open question here is whether or not long strings survive unusually long in higher dimensions. This will be put here to the test in the context of numerical simulations of strings. As a check on the numerical simulation and its equivalence to quantum strings, we compare the distribution of strings in thermal equilibrium studied for quantum strings in Refs. [8]

- [10], with the numerical results as a function of space dimension.

This article is organized as follows: in section 2 we descibe our 'experimental' setup (our numerical algorithm); in section 3 we study the thermodynamics of a string network as a function of string energy density and space dimensionality - we first explain what the prediction of quantum strings is and then compare it to what we find numerically; in section 4 we examine whether the lifetime of long strings depends on the space dimensionality; and in section 5 we state our conclusions.

\section{Description of the Model}

Before describing our discrete model we will briefly discuss the dynamics of continuous cosmic strings. Strings are represented [11] by a vector function $\vec{f}(\sigma, t)$, which satisfies

$$
\vec{f}^{\prime} \cdot \dot{\vec{f}}=0 ; \vec{f}^{\prime 2}+\dot{\vec{f}}^{2}=1
$$

where $\sigma$ is a parameter along the string; primes and overdots denote derivatives with respect to $\sigma$ and $t$, respectively. Equations (1) are gauge conditions on the

\footnotetext{
${ }^{2}$ The dimension 3 is the most likely direction to expand because it has the larger phase space.
} 
parametrization of the string worldsheet, so that the Nambu - Goto equation of motion in flat spacetime reduces to the wave equation

$$
\ddot{\vec{f}}-\vec{f}^{\prime \prime}=0
$$

(The Nambu-Goto action leading to the above equation is a good approximation for the motion of cosmic strings as long as their radius of curvature is larger than their width, but it is exact as far as classical fundamental strings are concerned.) The meaning of those gauge conditions is that a string segment $d \sigma$ moves perpendicular to itself, carrying an energy $\mu d \sigma$ ( $\mu$ denotes the mass per unit length of a string). Equation (2) admits the general solution

$$
\vec{f}(\sigma, t)=\frac{1}{2}[\vec{a}(\sigma-t)+\vec{b}(\sigma+t)]
$$

where $\vec{a}$ and $\vec{b}$ are arbitrary vectors on a unit sphere. As strings move they may exchange partners at intersection points, with an intercommuting probability depending on the string coupling[.

We will now describe the model we will use. It is based on an algorithm proposed by Smith and Vilenkin [4] to which we refer the interested reader for more details. Here we will briefly mention some of its main properties. In our 'experimental' setup, each string is represented by a discrete number of points on it, which are equally spaced in the parameter $\sigma$ by a separation $\delta$. We consider the segments of strings between neighbouring points to have all the same energy. In other words, points on strings are equally spaced in energy, meaning that the total energy of a string is proportional to the number of points by which it is represented. We choose as unit of energy the energy between neighbouring string points, while as unit of length and time the lattice spacing $\delta$. Strings are contained in a $D$-dimensional box of size $L$ with periodic boundary conditions, i.e., a torus. (Each string leaving the box reenters through the opposite face.) To evolve the sequence of points by which we

\footnotetext{
${ }^{3}$ In string theory the probability may change depending on the value of the dilaton.
} 
are representing strings, we define [4]

$$
\vec{v}(\sigma, t) \equiv \frac{1}{\delta}\left[\vec{f}(\sigma, t+\delta)-\frac{1}{2}[\vec{f}(\sigma+\delta, t)+\vec{f}(\sigma-\delta, t)]\right]
$$

as the discrete version of the string velocity $\dot{\vec{f}}(\sigma, t)$, becoming exact as $\delta \rightarrow 0$. Provided we know $\vec{f}$ and $\vec{v}$ at one instant, the equations

$$
\vec{f}(\sigma, t+\delta)=\frac{1}{2}[\vec{f}(\sigma+\delta, t)+\vec{f}(\sigma-\delta, t)]+\vec{v}(\sigma, t) \delta
$$

and

$$
\vec{v}(\sigma, t+\delta)=\frac{1}{2}[\vec{v}(\sigma+\delta, t)+\vec{v}(\sigma-\delta, t)]+\frac{1}{4 \delta}[\vec{f}(\sigma+2 \delta, t)-2 \vec{f}(\sigma, t)+\vec{f}(\sigma-2 \delta, t)],
$$

enable us to calculate both $\vec{f}$ and $\vec{v}$ at all times; Eqs. (5), (6) create an exact solution of Eq. (2) . ( $\vec{f}$ and $\vec{v}$ are determined on two interlocking but non-overlapping diamond lattices in the $(\sigma, t)$ plane.) Two consecutive points on a string segment determine a link of energy $2 \mu \delta$ moving at velocity $\vec{v}$. In this representation we assign position (but not velocity) to the points on the strings, while we assign velocity (but not position) to the links joining the string points. The discreteness of our model imposes a lower energy cutoff; we choose the cutoff parameter $E_{c}$ to be equal to 2, so that the shortest loops have just two links. Defining

$$
\vec{u}(\sigma, t) \equiv \frac{1}{2 \delta}[\vec{f}(\sigma+\delta, t)-\vec{f}(\sigma-\delta, t)]
$$

as the discrete version of $\vec{f}^{\prime}$, the gauge conditions, Eq. (11), take the discrete form

$$
\vec{u} \cdot \vec{v}=0 ; \vec{u}^{2}+\vec{v}^{2}=1
$$

and are preserved by the discrete evolution equations (Eqs. (5), (6)). We evolve all strings ahead by one timestep $\delta$ and subsequently allow them to intercommute, namely two strings passing through the same lattice site simply reconnect with probability $p$. We choose $p=1$ in this paper (but it would also be interesting for future work to allow this to vary with the value of the dilaton). We then proceed with 
the next timestep. An attractive feature of our model is that the algorithm for the string dynamics exactly integrates the equations of motion, while the intercommuting algorithm exactly preserves energy and momentum.

In this experimental setup a string state of energy $E$ will look like a random walk consisting of $E$ points on the lattice, which will have a physical length scaling like $\sqrt{E}$. In our experimental setup it is convenient to divide string states in two types: the long ones and the short ones. To study string thermodynamics we classify a loop as a short one if its energy is less than $E<L^{2}$ and long one if its energy is equal or greater than $E>L^{2}$. Note that since a typical configuration of a string is a random walk a long loop typically winds at least once around the toroidal universe. We have checked that the results we find are insensitive to the precise choice of the definition of short and long strings. To study the lifetime of the winding string modes, we employ a more restrictive criterion. At each timestep of the evolution of the string network, we calculate the extent of all loops in all directions. A loop will be defined as a long one if at least one of its extents exceeds $k L$, where $k$ is a numerical coefficent and $L$ stands for the size of the $D$-dimensional toroidal box.

\section{String Thermodynamics}

It is one of the basic facts about string theory that the degeneracy of string states increases exponentially with energy

$$
d(E) \sim \exp \left(\beta_{H} E\right)
$$

A simple consequence of this is that there is a maximum temperature which is $T_{\max }=$

$\beta_{H}^{-1}$, known as the Hagedorn temperature [12], [13], [14]. In the microcanonical ensemble the description of this situation is as follows: We put strings in a box with a certain total energy. Let us consider the energy density

$$
\rho=\frac{E}{L^{D}}
$$


Now for energies small enough, or more precisely for $\rho \ll 1$, the configuration of strings in equilibrium will be dominated by short strings (i.e., the massless modes in the quantum description). However as we increase the energy density more and more oscillatory modes of strings get excited. In particular, as shown in the work of Refs. [8] - 10], if we reach a critical density $\rho_{H}$ then long oscillatory string states begin to appear in the equilibrium state. The density at which this happens corresponds to the Hagedorn temperature. For any density $\rho>\rho_{H}$ the long oscillatory strings continue to be present in the equilibrium state and take up more and more of the total energy available. The description of the number of strings of various string energies for $\rho>\rho_{H}$ was worked out in Ref. [10] for fundamental strings in a box. The conclusion is as follows: There is a clear separation of string states which are long and oscillatory and the ones which are short. For short strings the number distribution in a given energy range goes as

$$
\frac{d n}{d E} \propto E^{-(1+D / 2)}
$$

Note that this means that there is a finite amount of total energy that the short strings can have. Let us denote this energy by $E_{H}=\rho_{H} L^{D}$. So all the excess energy $E-E_{H}$ goes to the production of long strings. The number distribution for long strings goes as

$$
\frac{d n}{d E}=\frac{1}{E}
$$

which means than the total number of long strings is roughly $\log \left(E-E_{H}\right)$. So typically the number of long strings grows very slowly with energy (in particular in our simulations we find that we only have typically a few of them at equilibrium). So in a situation with $\rho>\rho_{H}$ we have a few long strings which take up most of the energy of the system. The small loops behave like a 'background gas'.

There is an order parameter for the Hagedorn phase transition: Let $\tau_{l}(E)$ denote the lifetime of a long string of energy $E$; by lifetime we mean the time it takes for the long string to decay into short string states. Then for $\rho<\rho_{H}$ all the long strings 
decay and disappear from the thermal equilibrium state so that $\tau_{l}<\infty$. However for $\rho \geq \rho_{H}$ since long strings persist at equilibrium we have $\tau_{l}=\infty$. In particular, this means that there is an exponent $\alpha(E)$ that captures this transition, namely if we approach $\rho \rightarrow \rho_{H}$ from below, then

$$
\tau_{l}(E) \propto{\frac{\rho_{H}-\rho^{-\alpha(E)}}{\rho_{H}}}
$$

All of this is well defined for quantum string theory. However it would be nice if these features were also true in the classical domain of strings. Indeed, we will now see that the lattice model we described in the previous section has exactly the same features as expected for fundamental strings. The case of $D=3$ has already been studied in Ref. [15], which indeed shares the same thermodynamical properties as we described above. So the main aim of our simulations is twofold:

1-To check this correspondence for higher $D$ and in particular compute $\rho_{H}$ as a function of $D$. This is not a trivial statement and a priori it may have not been clear that string reconnections in this lattice model we are considering are frequent enough to even reach equilibrium. In particular, there is the question that, since the strings may have a hard time finding one another, there may be no dynamical way of reaching equilibrium in string theory in higher dimensions.

2-To Study the lifetime of long strings and its dependence on dimension. As discussed in the introduction, this issue was motivated by questions raised by string cosmology.

As a first step we would like to check whether the existence of a phase transition characterized by the appearenace of long loops is a generic property independent of the space dimensionality $D$. Secondly, we want to study the dependence of $\rho_{H}$ on the space dimensionality $D$. Since we expect that any dependence on $D$ will appear once we go from $D=3$ to $D=4$, and also due to computer limitations, we investigate how the equilibrium properties of a string system depend on the space dimensionality $D$, for $D=3,4$, or 5 . 
To study the equilibrium properties of the string network we perform computer simulations at various string densities. The initial states of those runs were chosen in the form of a 'loop gas', consisting of the shortest loops with randomly assigned positions and velocities. Each of these loops has just two 'degenerate' links. A degenerate link has [4] $\vec{u}=0$ and $|\vec{v}|=1$ (one component of $\vec{v}$ is \pm 1 and the other two zero); its end points are degenerate, $\Delta \vec{f}=0$. Such links are fully contracted and move at speed of light; they are a discrete version of the cusps that exist in continuous strings. The state of equilibrium should not depend on the choice of initial state. The loop gas was chosen both because its energy density is easily adjustable, and also because the Monte Carlo algorithm simulating the formation of strings at a phase transition [16] is only applicable for $D=3$. We ran our simulations in $D$-dimensional boxes of size $L$ from 10 to 50 for $D=3$; and $L$ from 10 to 20 for $D=4,5$.

Since, as we will discuss, we find that in accordance with the prediction of fundamental string equilibrium, there is a fixed maximum energy going to the creation of short loops for $\rho>\rho_{H}$, in order to compute the critical Hagedorn density $\rho_{H}$ we can consider any thermal equilibrium with $\rho>\rho_{H}$ and find $\rho_{H}$ from

$$
\rho_{H}=\rho x_{s}
$$

where $x_{s}$ denotes the ratio of lattice points occupied by short loops to the number of lattice points occupied by loops of all sizes (which is $N$ ) at equilibrium. (Note that $\rho \equiv N / L^{D}$.) The fact that $\rho_{H}$ thus obtained does not depend on which $\rho>\rho_{H}$ we start with, is a confirmation of the fact that beyond Hagedorn density all the energy goes to the creation of long strings.

We have indeed found a sharp change of behaviour at $\rho \sim \rho_{H}$. For $\rho<\rho_{H}$, there are no long loops, their energy density, $\rho_{l}$, is zero. For $\rho>\rho_{H}$, the energy density in short loops, $\rho_{s}$, is constant and equal to $\rho_{H}$, while

$$
\rho_{l}=\rho-\rho_{H} \quad\left(\text { for } \rho>\rho_{H}\right) .
$$


Figure 1 shows the energy density in short loops, $\rho_{s}=\rho-\rho_{l}$, as a function of the string density $\rho$. The value of $\rho_{H}$ depends on the size of the smallest allowed loops. If the smallest allowed loops consist of only two points, then the values we obtain for the critical string density, performing runs at rather high string densities, are:

$$
\rho_{H}= \begin{cases}0.172 \pm 0.002 & \text { for } D=3 ; \\ 0.062 \pm 0.001 & \text { for } D=4 ; \\ 0.031 \pm 0.001 & \text { for } D=5\end{cases}
$$

We can now study the size distribution of short loops at the high-energy regime, once equilibration is established. We denote by $d n$ the number of loops with energies from $E$ to $E+d E$. Figure 2 shows the energy distribution of short loops in the highenergy density regime, as a function of space dimensionality. We have checked that these distributions are independent of the particular value of $\rho\left(\rho>\rho_{H}\right)$ for a given $D$. We find that the size distribution of short loops is well defined by a line

$$
\frac{d n}{d E} \sim E^{-(1+D / 2)}
$$

where the space dimensionality $D$ was taken equal to 3,4 , or 5 . This is what was indeed expected from analytical string results. The statistical errors indicate a slope equal to $-(1+D / 2) \pm 0.2$. Above the Hagedorn energy density the system is characterized by a scale invariant distribution of short loops and a number of long loops with a distribution that is not scale invariant.

The evolution of a network of loops reveals the existence of two relaxation times: a relaxation time $t_{r e l}$ defined as the time by which the ratio $x_{s}$ equilibrates and a relaxation time $\tau_{\text {rel }}$ defined as the time by which the slope in the size distribution of short loops reached the value $-(1+D / 2)$ and remained constant thereafter. It is clear that while $t_{r e l}$ has a meaning for any value of string density, $\tau_{r e l}$ is only meaningful for string densities above the critical one $\rho_{H}$. Both these relaxation times are of the same order of magnitude, which is at most about 100 evolution timesteps. The relaxation time seems to be independent of either the string density $\rho$ or the space dimensionality $D$. 


\section{Lifetime of Long Strings}

Our next goal is to study the lifetime of long loops (which represent the winding modes) as a function of space dimensionality and string energy density. As one expects, in the high energy density regime, in other words above the Hagedorn energy density, the long loops do remain indefinitely. Around the critical energy density $\rho_{H}$, the lifetime of long loops fluctuates a lot. The interesting regime is the low energy density one, namely the case below the Hagedorn energy density. To address the issue of the lifetime of long string loops in the low energy density regime, as a function of space dimensionality, we basically pursue the following approach:

In a $D$-dimensional toroidal box of size $L$, we create a 'loop gas' consisting of $s-$ point loops $(s>2)$, at a string energy density greater than the critical one, but below the value of $\rho_{H}$ corresponding to $s=2$ (given by Eq. (16) for $D=3,4,5$ ). [Note that,

if for example $D=3$, the critical string density goes roughly as $s^{-2}$.] The energy of these loops is $E_{s}$. We evolve the system until timestep $t_{s}$, under the condition that the shortest loops which are allowed to be chopped off, must have at least an energy $E_{s}$. Since we are in the high-energy density regime, once the system reaches equilibrium we expect the existence of very long string modes, winding a number of times around the torus. During the subsequent evolution timesteps, $t>t_{s}$, we freeze out short loops up to an energy $E_{f}$, so that the rest of the network has an energy density below the Hagedorn energy density corresponding to $E_{c}=2$. We then drop the energy cutoff to 2 , which leads to an increase of the critical string density $\rho_{H}$, reaching the values given in Eq. (16). Now we are in the low-energy density regime and long string loops would be expected to decay in order for equilibrium to be maintained.

In our simulations we create a network of twelve- and two-point loops at high enough energy densities, in boxes of size $L$ from 10 to 50 for $D=3$ and $L$ from 10 to 20 for $D=4,5$. We evolve the loop string networks until equilibrium is reached and 
long string states winding around the toroidal box are formed, allowing loops of all sizes to reconnect, but forbiding the creation of loops having less than 12 points. We then freeze small loops, up to usually six- to eight-point loops, so that the string density drops below the critical one for $E_{c}=2$. We subsequently set the cutoff $E_{c}$ to be indeed 2 and we see that while for $D=3$ the long string loops decay after a few timesteps, for $D=4$ or $D=5$, long loops representing the winding states persist even after a large number of evolution timesteps. Figure 3 shows 'snapshots' of the 3- $D$ string network (a) at $t=0$, when the system consists of twelve- and two-point loops; (b) at $t=t_{s}$, when the system consists of short loops and long ones, winding around the box; and (c) at a relaxation time, showing the decay of all winding string modes.

There are two other approaches which we followed in order to study the lifetime of long string modes, and both of them support the previously stated result. In what follows, we will briefly describe those two methods.

1-Our initial configuration consists of a loop gas at a string density $\rho$ above the critical value $\rho_{H}$, i.e.,

$$
\rho=\alpha \rho_{H}(\alpha>1)
$$

We evolve this system and, once equilibrium is reached, the density of long strings must be

$$
\rho_{l}=(\alpha-1) \rho_{H} .
$$

We are interested in the cases where $\rho_{l}$ is smaller than $\rho_{H}$. (We have done various runs for $D=3,4$, or 5 to verify the above statement.) We can now disregard the short loops and consider the network of only long loops. As a result of the imposed periodic boundary conditions, the total winding number is zero. We thus evolve a very energetic string or a network of a few very energetic strings in the low energy density regime in a $D$-dimensional box, where $D=3,4$, or 5 . The lifetime of winding modes is the inverse of the slope of the $E_{l}$ versus time $t$ curve, 
where $E_{l}$ denotes the number of lattice points (i.e., energy) occupied by long loops. Our qualitative results indicate a strong dependence on the space dimensionality $D$. While for $D=3$ the lifetime of long loops is rather short (much shorter than the relaxation time), as the space dimensionality increases (i.e., $D>3$ ), the lifetime of the very energetic loops increases dramatically; winding modes remain 'indefinitely'. At this point, one might wonder whether the rather short lifetime of long loops, when the space dimensionality $D=3$, is a result of the shape of those loops. In other words, it might be that the very energetic loops survive for a short time just because they are locally rather rough. To check this possibility, we have studied the Hausdorff dimension of loops. We estimate the Hausdorff dimension $\nu$ as follows: we calculate the average distance, $d$, between two points on the loop, as a function of the length, $l$, along the loop. The length was found by connecting the points of the loop by straight lines, and the averaging was done over a large number of loop segments having lengths in specified narrow intervals (of unit width). The Hausdorff dimension $\nu$ is defined as

$$
\nu \equiv \log (l) / \log (d)
$$

Our numerical simulations indicate that initially the energetic strings are of the form of random walks $(\nu=2)$ independently of space dimensionality $D$. This is exactly what we were expecting to find: since the very energetic strings were the outcome of the evolution of a loop gas above the Hagedorn energy density, their shape should indeed be approximately Brownian. Now, as this low-energy density (short loops have been disregarded) network of winding modes evolves, these long strings become almost straight (as it was indeed expected). We thus conclude that the disappearance of winding modes in the case of $D=3$ space dimensionality is not the result of any particular local roughness of the loops configurations. The 'weak' point of this approach is that the network of long string states, which was created evolving the initial high-energy density loop gas, consisted mainly of string loops 
winding only a few times around the torus.

2-In a $D$-dimensional toroidal box of size $L$ we create a network of long string states winding a large number of times around the torus. We create this system in such a way that for each pair of opposite faces of the box, if a string going from face $j$ towards face $j^{\prime}$, cuts $n_{j}$-times this face $j^{\prime}$, then there must be another string state, having the opposite direction, which cuts also $n_{j}$-times the face $j$. To this string configuration, composed of a number of long string states winding many times around the torus, we add zero-length links, so that we are always in the low-energy density regime. The evolution of such a string network shows that long string states decay only if the space dimensionality of the torus is $D=3$.

The persistence of long loops at string energy densities below the critical one, when the space dimensionality is $D>3$, supports the proposed cosmological scenario by Brandenberger and Vafa [5], which attempts to explain the space dimensionality and to avoid the initial singularity by means of string theory. According to their scenario, the universe starts as a compact space in a high number of space dimensions. Its expansion is slowed down due to the existence of winding modes (their energy grows linearly with $L$ ), which being unable to find one another, fail to annihilate and fall out of equilibrium. The universe will contract until the momentum modes slow the contraction, as the universe reached a size below the Planck scale (a consequence of the target space duality). As a result the universe will undergo an oscillatory period, avoiding both the initial singularity and the divergence of the temperature at the Big Bang. At some point, fluctuations might accidentally lead to a 3-dimensional expansion, leading to the annihilation of winding modes and the irreversible birth of the 3-dimensional decompactified universe [5]. As those authors suggest [5], in exactly $(3+1)$-spacetime, the 2-dimensional world-sheets of the winding modes can intersect, unwinding themselves. However, the fact that the maximum spacetime dimensionality required in order to maintain thermal equi- 
librium is 4 , does not explain why the space dimensionality is 3 and not a lower one. A suggestion to resolve this issue, which however has not been worked out, was proposed in Refs. [5], [17].

\section{Conclusions}

In this paper we have studied via numerical experiments some classical aspects of fundamental strings. Our aim was basically to test a scenario of string cosmology proposed by Brandenberger and Vafa [5], based on the heterotic string theory in the space of a 9-dimensional toroidal universe of Planckian size and one time dimension. The basic idea of the model is that such a small universe underwent a long period of oscillations, until finally three out of nine dimensions began to expand leading to our present universe. Performing a series of numerical simulations based on a simple and exact algorithm, we studied the thermal properties and the equilibration process of a string network in a toroidal universe, and in particular its dimension dependence. Our results are in a full agreement with those found for quantum strings. At the low-energy density regime the string network is dominated by the shortest allowd string loops, which represent the string momentum modes. As the energy density increases, a phase transition takes place, characterized by the appearance of long string loops winding around the torus; these represent the winding string modes. The phase transition is a generic property independent of the space dimensionality. However the space dimensionality plays a crucial role for the lifetime of the long

string loops. As we found, only if the space dimensionality is equal to three, long winding string modes decay in the low-energy density regime, while these modes being unable to find one another, fail to annihilate and persist indefinitely in a higher dimensional space.

\section{Acknowledgement}


It is a pleasure to express my thanks to Cumrun Vafa, for raising my interest in this problem, for a number of stimulating discussions which we had and for his invitation to the Lyman Laboratory at Harvard University, where part of this work was done. I would also like to thank both Alex Vilenkin and Robert Brandenberger for their suggestions, comments and helpful discussions.

\section{References}

[1] Y. Nambu, in proceedings of Int. Conf. on Symmetries and Quark Models, (Wayne State University) (1969); Lectures at the Copenhagen Summer Symposium (1970).

[2] T. Goto, Prog. Theor. Phys. 46, 1560 (1971).

[3] P. Shellard, Nucl. Phys. B283, 624 (1987).

[4] A.G. Smith and A. Vilenkin, Phys. Rev. D36, 990 (1987).

[5] R. Brandenberger and C. Vafa, Nucl. Phys. B316, 391 (1989).

[6] K. Kikkawa and M. Yamasaki, Phys. Lett. B149, 357 (1984); V. Nair, A. Shapere, A. Strominger and F. Wilczek, Nucl. Phys. B287, 402 (1987); P. Ginsparg and C. Vafa, Nucl. Phys. B289 414 (1987); B. Sathiapalan, Phys. Rev. Lett. 58, 1597 (1987); A.A. Tseytlin, Mod. Phys. Lett. A6, 1721 (1991).

[7] A.A Tseytlin and C. Vafa, Nucl. Phys. B372, 443 (1992).

[8] M.J. Bowick and L.C.R. Wijewardhana, Phys. Rev. Lett. 54, 2485 (1985).

[9] D. Mitchell and N. Turok, Phys. Rev. Lett. 58, 1577 (1987); Nucl. Phys. B294, 1138 (1987).

[10] N. Deo, S. Jain and C. Tan, Phys. Rev. D40, 2626 (1989); Phys. Lett. B220, 125 (1989). 
[11] P. Goddard, J. Goldstone, C. Rebbi and C.B. Thorn, Nucl. Phys. B56, 109 (1973).

[12] R. Hagedorn, Nuovo Cimento Suppl. 3, 147 (1965).

[13] R.D. Carlitz, Phys. Rev. D5, 3231 (1972).

[14] S. Frautschi, Phys. Rev. D3, 2821 (1971).

[15] M. Sakellariadou and A. Vilenkin, Phys. Rev. D37, 885 (1988).

[16] T. Vachaspati and A. Vilenkin, Phys. Rev. D30, 2036 (1984).

[17] H. Câteau and K. Sumiyoshi, Phys. Rev. D 46, 2366 (1992). 


\section{Figure Captions}

Fig. 1a: The energy density of short loops, $\rho_{s}=\rho-\rho_{l}$, as a function of the total string energy density $\rho$. Strings are moving in a 3-dimensional torus. The smallest allowed loops consist of only two points. There is a sharp change of behaviour at $\rho_{H} \sim 0.170$.

Fig. 1b: The energy density of short loops, $\rho_{s}=\rho-\rho_{l}$, as a function of the total string energy density $\rho$. Strings are moving in a 4-dimensional torus. The smallest allowed loops consist of only two points. There is a sharp change of behaviour at $\rho_{H} \sim 0.062$.

Fig. 1c: The energy density of short loops, $\rho_{s}=\rho-\rho_{l}$, as a function of the total string energy density $\rho$. Strings are moving in a 5 -dimensional torus. The smallest allowed loops consist of only two points. There is a sharp change of behaviour at $\rho_{H} \sim 0.031$.

Fig. 2: Energy distributions of short loops in the high-energy density regime, for strings moving in a $D$-dimensional torus with $D=3,4,5$, as we go from right to left respectively. These distributions do not depend on the particular value of $\rho$ $\left(\rho>\rho_{H}\right)$ and are well fitted by $d n / d E \sim E^{-(1+D / 2)}$.

Fig. 3a.1: Snapshot of the initial state of a string gas (a) in a 3-dimensional torus. It consists of $12-$ and 2-point loops in a box of $L=30$, where 5400 points are occupied by strings. 
Fig. 3a.2: Snapshot of the string system (a) at $t=60$. Only the 2 long strings having energies equal to 417, 1113 respectively, are shown. Until this timestep the smallest allowed energy cutoff is $E_{c}=12$.

Fig. 3a.3: Snapshot of the string system (a) at the relaxation time $t=109$. There are no long string modes and they do not appear later on. The cutoff was dropped to $E_{c}=2$, while $E_{f}=6$.

Fig. 3b.1: Snapshot of the initial state of a string gas (b) in a 3-dimensional torus. It consists of $12-$ and $2-$ point loops in a box of $L=20$, where 2200 points are occupied by strings.

Fig. 3b.2: Snapshot of the string system (b) at $t=100$. Only the 4 long strings having energies equal to 168, 238, 255, 471 respectively, are shown. Until this timestep the smallest allowed energy cutoff is $E_{c}=12$.

Fig. 3b.3: Snapshot of the string system (b) at the relaxation time $t=153$. There are no long string modes and they do not appear later on. The cutoff was dropped to $E_{c}=2$, while $E_{f}=8$. 
This figure "fig1-1.png" is available in "png" format from: http://arxiv.org/ps/hep-th/9511075v1 
This figure "fig2-1.png" is available in "png" format from: http://arxiv.org/ps/hep-th/9511075v1 
This figure "fig3-1.png" is available in "png" format from: http://arxiv.org/ps/hep-th/9511075v1 
This figure "fig4-1.png" is available in "png" format from: http://arxiv.org/ps/hep-th/9511075v1 
This figure "fig1-2.png" is available in "png" format from: http://arxiv.org/ps/hep-th/9511075v1 
This figure "fig2-2.png" is available in "png" format from: http://arxiv.org/ps/hep-th/9511075v1 
This figure "fig3-2.png" is available in "png" format from: http://arxiv.org/ps/hep-th/9511075v1 
This figure "fig4-2.png" is available in "png" format from: http://arxiv.org/ps/hep-th/9511075v1 
This figure "fig1-3.png" is available in "png" format from: http://arxiv.org/ps/hep-th/9511075v1 
This figure "fig2-3.png" is available in "png" format from: http://arxiv.org/ps/hep-th/9511075v1 